\title{
A Software Development Toolkit for Real-Time Synchrophasor Applications
}

\author{
Luigi Vanfretti ${ }^{1}$, Vemund H. Aarstrand ${ }^{2}$, M. Shoaib Almas ${ }^{1}$, Vedran S. Perić ${ }^{1}$ and Jan O. Gjerde ${ }^{2}$
}

\begin{abstract}
${ }^{1}$ Abstract - This article presents a software development toolkit for Wide Area Monitoring Systems. By using this development toolkit, a researcher is able to manipulate synchrophasor data in the LabView environment, which enables fast software prototyping and testing. This toolkit makes full scale testing in real-time easier for researchers, liberating them of complex and time consuming synchrophasor data handling. The toolkit exploits the IEEE C37.118.2 - 2011 protocol making it independent of any specific equipment and their manufacturers. An application of the development kit is demonstrated in a laboratory environment with a specially designed experimental setup composed of a real-time digital simulator and four phasor measurement units (PMUs).
\end{abstract}

Keywords - Wide Area Monitoring Systems, software development toolkit, PMU applications, real time hardware-inthe-loop simulation

\section{INTRODUCTION}

Wide Area Monitoring Systems (WAMS) are one of the key elements needed for intelligent operation of power systems and are enablers for Smart Grids at the transmission level [1] [2]. These systems, which have been used across the world by different Transmission System Operators (TSOs), successfully contribute to operational enhancement of power systems [3]. In addition, WAMS performances and applications are continuously improving as a result of intensive ongoing research and development. A lot of research is being carried out to exploit synchrophasor in applications like power oscillation detection and damping [4] [5], voltage stability monitoring [6], power system protection [7] [8], etc.

Generally, there are three main active areas of research and development in this field:

1. Design and development of a new generation of Phasor Measurement Units (PMUs);

2. Development of WAM communication subsystems;

3. Design of advanced synchrophasor-based algorithms for monitoring and control.

Even though researchers are making significant progress in these areas, a rising challenge is testing of the integral WAMS solutions in real-time. To test such WAMS solutions, it is necessary to:

1. Feed physical PMUs with proper (real-life) input signals

2. Connect PMU outputs to phasor data concentrators (PDCs) with arbitrary configuration.

\footnotetext{
${ }^{1}$ L. Vanfretti, M. S. Almas and V. Peric are with KTH Royal Institute of Technology, Stockholm, Sweden. Corresponding author: luigiv@kth.se

2 L. Vanfretti, V. H. Aarstrand and J. O. Gjerde are with Stattnet SF, Research and Development, Oslo, Norway.
}

3. Stream the data out from the PDCs and convert it to a data model suitable for real-time analysis and development of new applications.

These tasks require carefully designed laboratory environments [9] with utility tools provided by vendors whereas developed applications are implemented in low-level program languages like $\mathrm{C}, \mathrm{C}++$, etc. Consequently, researchers need to devote large efforts developing mechanisms that allow them to use PMU measurements, e.g. PMU data extraction. These tasks are especially difficult in the case where equipment and concentrators are provided by different vendors.

This paper presents a software development toolkit developed by Statnett SF (the Norwegian Transmission System Operator) in collaboration with the Smart Transmission Systems Laboratory (SmarTS Lab.) at KTH Royal Institute of Technology for facilitating research, fast prototyping and testing of real-time synchrophasor applications. The development toolkit enables the usage of high-level programing languages such as Labview, regardless of the equipment used and its manufacturer, thus providing platform independency for research and development. This property enables researchers to be more focused on developing synchrophasor applications and not on platformspecific implementation issues. The development toolkit is capable of connecting to an arbitrary number of PMUs or PDCs compliant with the IEEE C37.118.2 - 2011 protocol [10]. The development toolkit reads and stores real-time data in a configurable buffer. Currently, the content of the buffer is accessible in the Labview environment through a standard Labview function control (VI), and accepts connections from a single PDC. Future versions of the toolkit, which are currently under development, will also allow these functionalities directly in MATLAB and arbitrary connections to multiple PMUs or PDCs.

The reminder of this paper is organized as follows: Section_II provides information about the general data transfer architecture of wide-area monitoring systems. Section III presents the architecture of the development toolkit. Section IV focuses on end-to-end testing of the software by utilizing synchrophasor streams from both real PMUs in the field and HIL simulation streams from PMUs interfaced with Opal-RT's eMEGAsim real-time simulator. The development of a real-time mode estimation application based on the software development toolkit is discussed in Section $\mathrm{V}$ as an example of how the toolkit allows making custom applications by researchers. Finally, conclusions are drawn in Section VI. 


\section{WAM SYSTEM ARCHITECTURE}

The development of PMUs and their capability to accurately measure dynamics of power system enabled an implementation of advanced monitoring and control applications [11] [12]. PMUs stream out synchronized phasor measurements at high sampling rates (typically $30 \mathrm{~Hz}, 50 \mathrm{~Hz}$ or $60 \mathrm{~Hz}$ ) which are synchronized to an absolute time reference using Global Positioning Satellites (GPS) clocks. A large number of PMUs are currently being deployed in transmission networks to observe the status of the power system at a very high resolution. Vendors of protection relays have already incorporated the functionality of PMUs in their equipment, enabling their devices to operate both as a protection relay and a PMU simultaneously.

The data from the PMUs is received by Phasor Data Concentrator (PDC). A PDC can communicate with multiple PMUs and other PDCs to acquire synchronized phasor measurements and time-align them. In addition, it also provides features like archiving data for post-fault analysis and monitoring the status of all the PMUs and PDCs connected to it. Similarly, the PDCs can send their output streams to other higher level PDCs (which may have more processing and storage capability) to finally get a single output stream which can be used by the power system operators in the control center to visualize the status of the power system. Fig. 1 shows an example of a conventional WAMS architecture with two layers of PDCs where top layer (Super) PDC sends a single PDC stream to the control center.

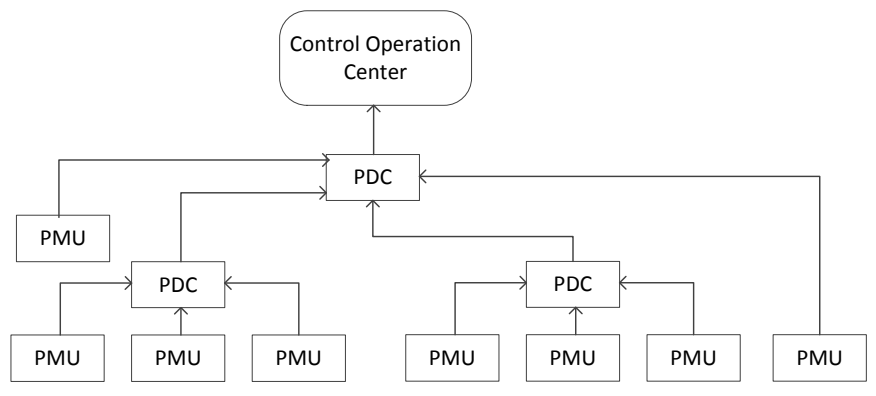

Figure 1. Typical Layout of the communication between PDCs

In order to develop applications for monitoring and control based on synchrophasor measurements, it is important to have real-time access to the individual quantities (phasor/analog/digital) of each PMU, which are wrapped inside the real-time PDC stream.

\section{LABVIEW-BASED SOFTWARE DEVELOPMENT TOOLKIT}

The proposed Labview software development toolkit (SDK) presented in this article enables easy real-time access to PMU and PDC streams. The Labview platform is selected because it provides easy integration with different hardware equipment as well as intuitive graphical programing language (G language) which supports integration with MALTAB.

The SDK is named "PMU Recorder Light" (PRL). The PRL has two major components as listed below:
1. Data Collector. This component reads the data from the PDC/PMU and stores them in configurable buffers.

2. Data Extractor. This is a collection of functions (VIs ${ }^{2}$ ) that allows the user to access the buffers and queues in the PRL. It reads the data from the buffers and provides the user with control over the data streams in a form suitable for further processing (i.e. as a signal data type in Labview).

The general architecture of the SDK is shown in Fig. 2.

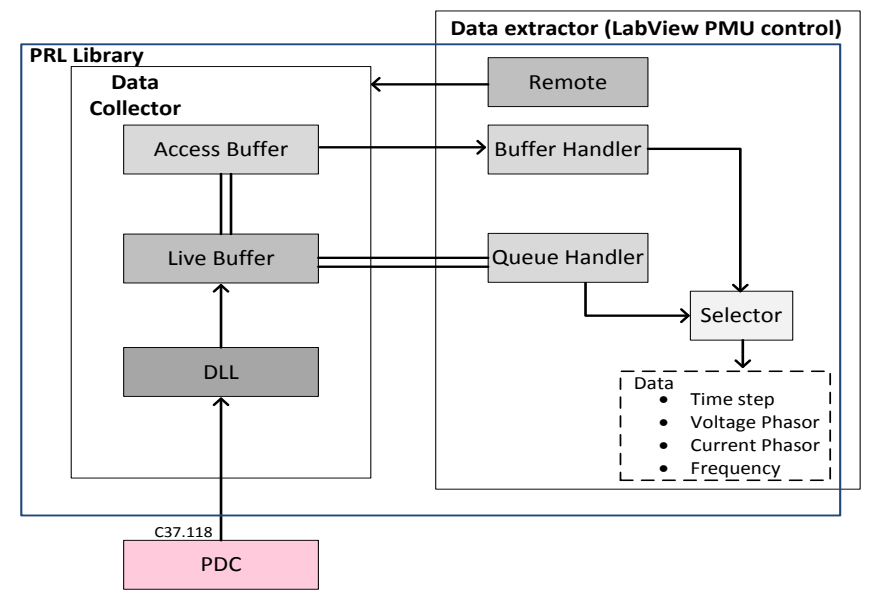

Figure 2. Major Components of the PRL

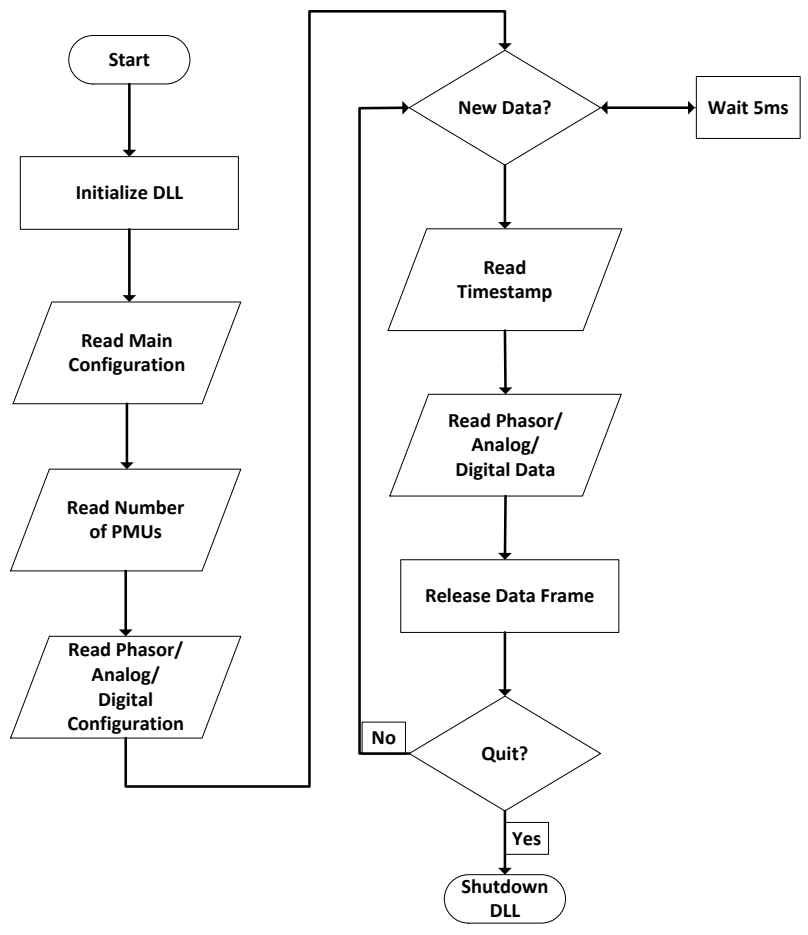

Figure 3. Flowchart for DLL Data Access

\section{A. Data Collector}

The Data Collector uses a Dynamic-link library (DLL) developed in the $\mathrm{C}$ programming language to connect to the PDC stream via the IEEE C37.118.2 protocol. In addition, the DLL reads the configuration data of the PDC stream, such as channel names, scaling and number of measured signals by

\footnotetext{
${ }^{2}$ VI's (Virtual Instruments) are the Labview term for internal functions.
} 
type (analog, phasors or digital signals). The algorithm of the DLL functionality is shown in Fig. 3.

The incoming data from the DLL are stored in the Live Buffer. When the data buffer is full, the data is put in a queue that sends data to the Access Buffer, the data then can be received by a custom user application using the Queue Handler. The Access Buffer size decides on the amount of data history to be kept in memory, and the data that can be read from the buffer in the Custom Application via the Buffer Handler.

\section{B. PRL to PDC Connection Handling}

The main benefit of the software development toolkit is that it exempts a developer of complicated synchrophasor data handling. Instead, the developer is required only to set the appropriate PRL to PDC connection parameters such as PDC ID, PDC host address and the port number to connect to the PDC stream (Fig. 4).

After the PDC stream is configured correctly in the PRL (Fig. 4), it is possible to monitor different parameters of the connection such as processing power used on incoming data from the PDC $(\%)$, processing power used to write data to storage buffer (\%), empty data ratio (\%), LEDs for showing successful connection with the PDC and toggle switch for options such as bad data removal. The graphical user interface with these features is shown in Fig. 5.

\section{Data extractor - LabView PMU control}

Once the connection is established, the PRL's Data Selector Tool allows the user to select data of interest and to use and/or visualize those (Figs. 6, 10). These data, which are initially stored in the access buffer, are imported into the user's Labview application by the Buffer Handler VI. This VI has 4 inputs and 7 outputs as shown in Fig. 6. By using the Buffer Handler, a developer has the possibility to extract data from the access buffer. It is possible to read all data from the buffer ("Get Full Buffer?" flag from Fig.6) or only data received after the previous reading of the buffer (to distinguish newly arrived data, a time stamp of the previous access must be remembered form the "Time Stamp In" input). At the output the user has access to the three groups of signals as it is shown in Fig. 6 (phasor, analog and digital signals).

\section{END-TO-END TESTING OF THE SDK}

\section{Experimental setup}

In order to validate the SDK and to test the integral WAMS system, three PMUs from SEL (one SEL-487E and two SEL-421), and one PMU from ABB (RES-521) were configured to form a WAMS system. These four PMU streams were sent to Phasor Data Concentrator (SEL-5073). Finally, two similar outputs were configured from the PDC. One PDC stream was sent to PRL and other PDC stream was directed to SEL Synchrowave Central (SEL-5078-2) which is a visualization tool for synchrophasors data. SEL PMUs were connected to the analog outputs of Opal-RT's eMEGAsim real-time simulator through Megger Smrt-1 Amplifiers. These amplifiers were tuned to amplify the low level voltages and currents from RTS to $30 \mathrm{~V}$ and $1 \mathrm{~A}$ respectively. The ABB PMU was connected directly to the real distribution network while SEL PMUs were connected to the Real-Time Simulator. Fig. 7 shows the overall deployment of PMUs in the laboratory facility and the test scenario layout used to validate the PRL software. The SEL PMUs were configured to stream out only positive sequence voltages and currents. The ABB PMU connected to the distribution grid, was streaming only positive sequence voltage.

Fig. 8 shows the single line diagram of the test system modeled in MATLAB/Simulink SimPowerSystem (SPS). This model was executed in real-time using the Opal-RT real time simulator. The voltages and currents of the simulated model were configured to the analog outputs of the simulators which were connected to the PMUs through the amplifiers.

The first step in validating the software results is to compare the number of PMUs, their respective phasors/analog/digital channels and their names. With this PDC stream, the results from SEL 5078-2 are shown in Fig. 9. It shows that the PDC comprises of 4 PMUs namely Differential (SEL-487E), Distance Top (SEL-421), Distance Bottom (SEL-421) and KTHLAB (ABB RES-521). All the SEL PMUs are streaming out positive sequence voltage and current phasors while the ABB PMU is streaming out only positive sequence voltage. With the same PDC stream, PRL software shows exactly the results which are shown in Fig. 10.
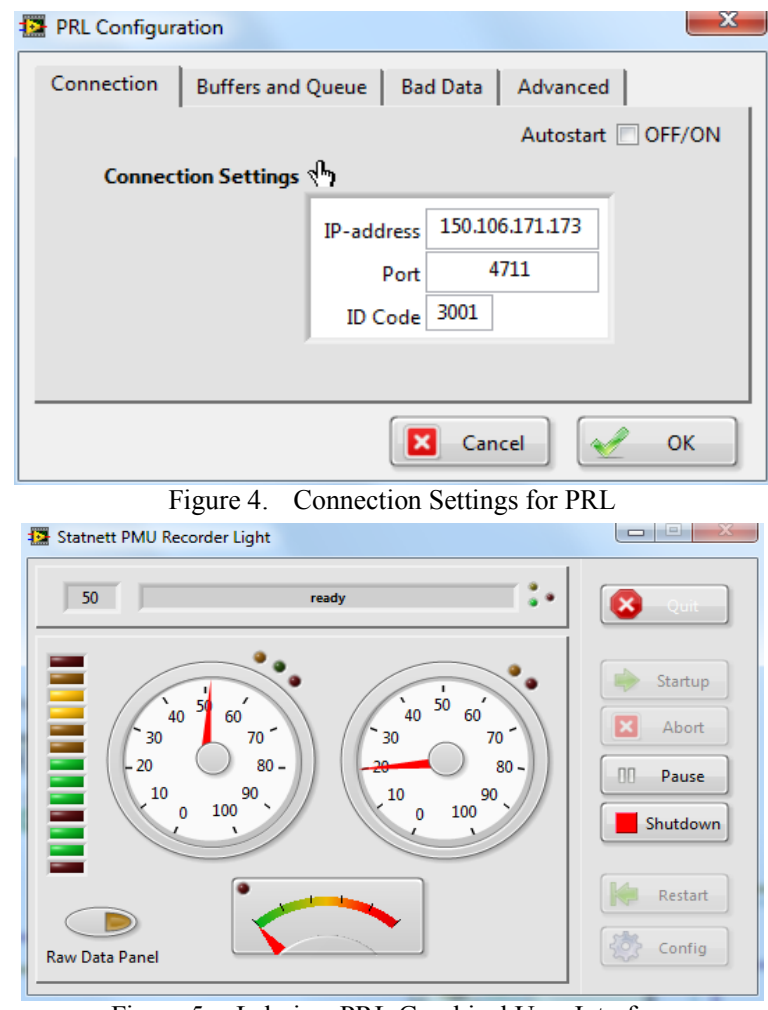

Figure 5. Labview PRL Graphical User Interface

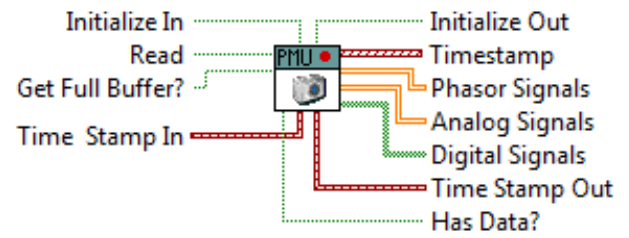

Figure 6. Labview PMU Control 


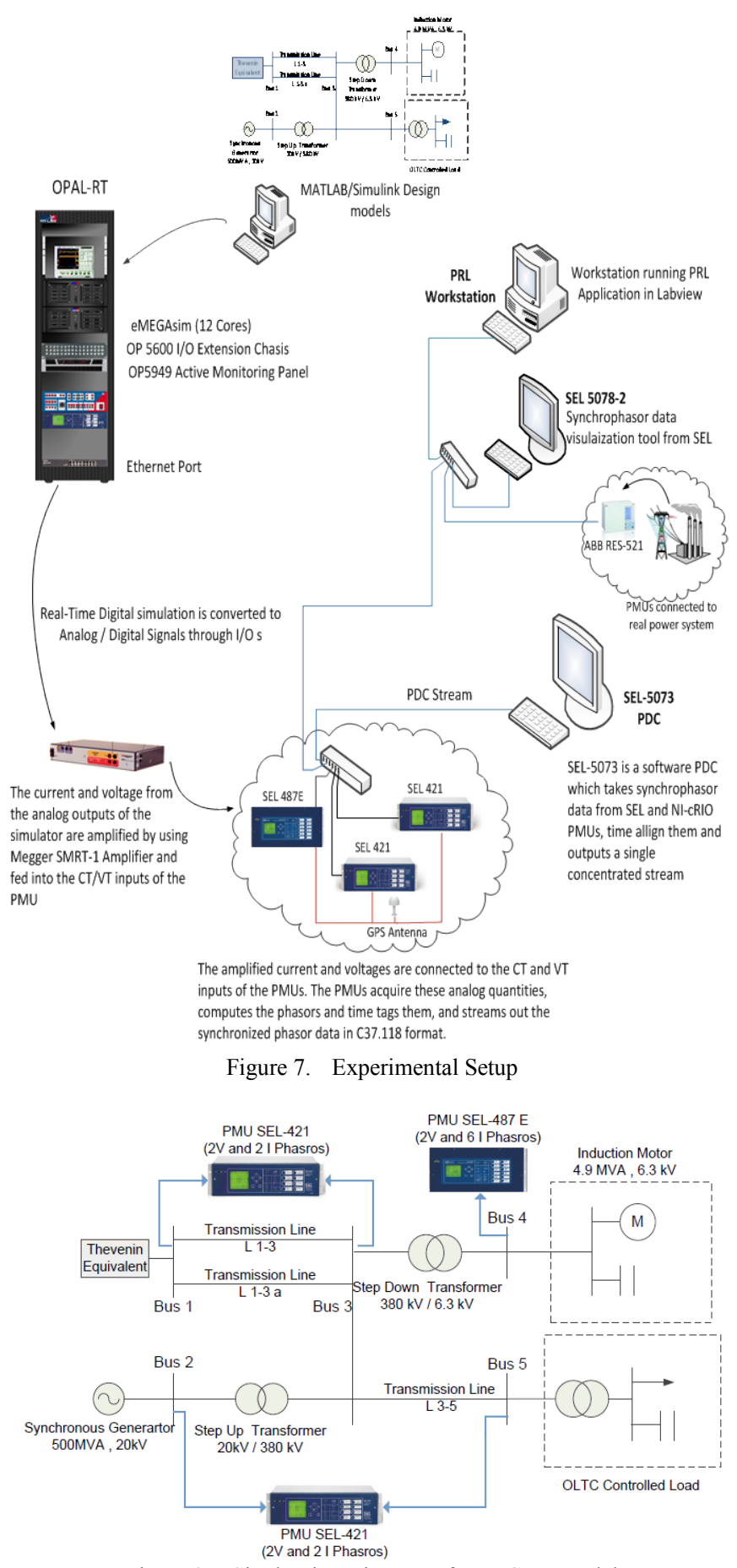

Figure 8. Single Line Diagram of Test Case Model

\begin{tabular}{|c|c|c|c|c|c|c|c|}
\hline \multicolumn{3}{|l|}{ Available Tags } & \multicolumn{5}{|c|}{ Filter } \\
\hline PMU & Tag & Description & Data Rate & Units & Type & Quantity T & Phase \\
\hline Differential & V1ZPM & & 50 & lv & Phasor & Voltage & \\
\hline Differential & I115PM & & 50 & A & Phasor & Current & \\
\hline Differential & IITPM & & 50 & A & Phasor & Current & \\
\hline Distance Bottom & VIYPM & & 50 & v & Phasor & Voltage & \\
\hline Distance Bottom & V1ZPM & & 50 & $\mathrm{v}$ & Phasor & Voltage & \\
\hline Distance Bottom & I1WPM & & 50 & A & Phasor & Current & \\
\hline Distance Top & VIYPM & & 50 & $\mathrm{v}$ & Phasor & Voltage & \\
\hline Distance Top & I1WPM & & 50 & A & Phasor & Current & \\
\hline Distance Top & V1ZPM & & 50 & $\mathrm{v}$ & Phasor & Voltage & \\
\hline Distance Top & IIXPM & & 50 & A & Phasor & Current & \\
\hline KTHLAB & EMLAB & Positive Sequence Voltage Terminal 1 & 50 & v & Phasor & Voltage & 1 \\
\hline
\end{tabular}

Figure 9. Results from SEL 5078-2 (Synchrowave Central Software)

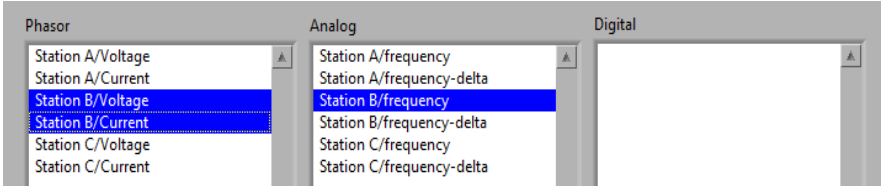

Figure 10. Results from PRL Data Selector Tool with same PDC Stream

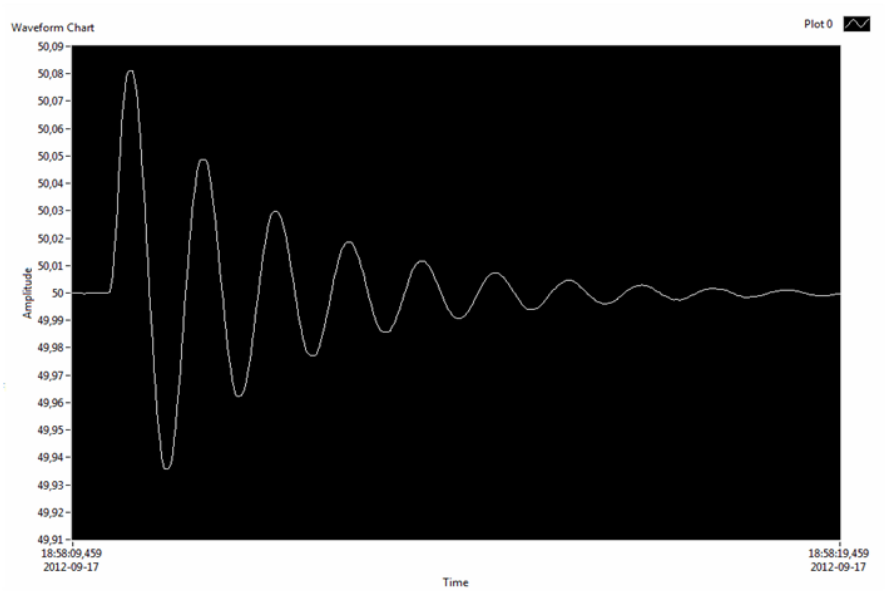

(a) Frequency Disturbance translated by PRL

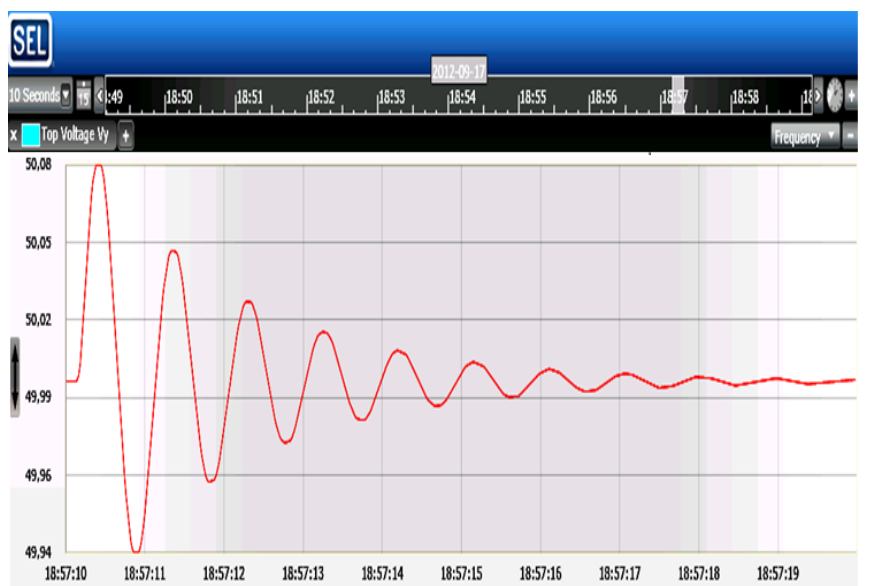

(b) Frequency Disturbance translated by SEL Synchrowave

Frequency disturbance monitored via the PRL and the SEL Synchrowave Central (SEL-5078-2)

For the validation of the software, the test model in Fig. 7 was executed in real-time and subjected to a disturbance. At a particular instant, the mechanical input power of the synchronous generator was increased to simulate a frequency disturbance. The voltages at all the buses and the frequencies as seen by the PMUs (which are coupled with the analog outputs of Opal-RT simulator via amplifiers) were analyzed. The analysis shows that the data obtained from the PRL are identical to the results with SEL 5078-2. Fig. 11(a) and Fig. 11(b) show the frequency at Bus 5 as captured by PMU SEL-421 and translated by both software. In order to further validate the accuracy of the PRL, the magnitude of the positive sequence voltage at all buses of the test case (executing in real time) were compared with SEL Synchrowave Central (SEL 5078-2). The results are shown in Fig. 12(a) and Fig 12(b). 


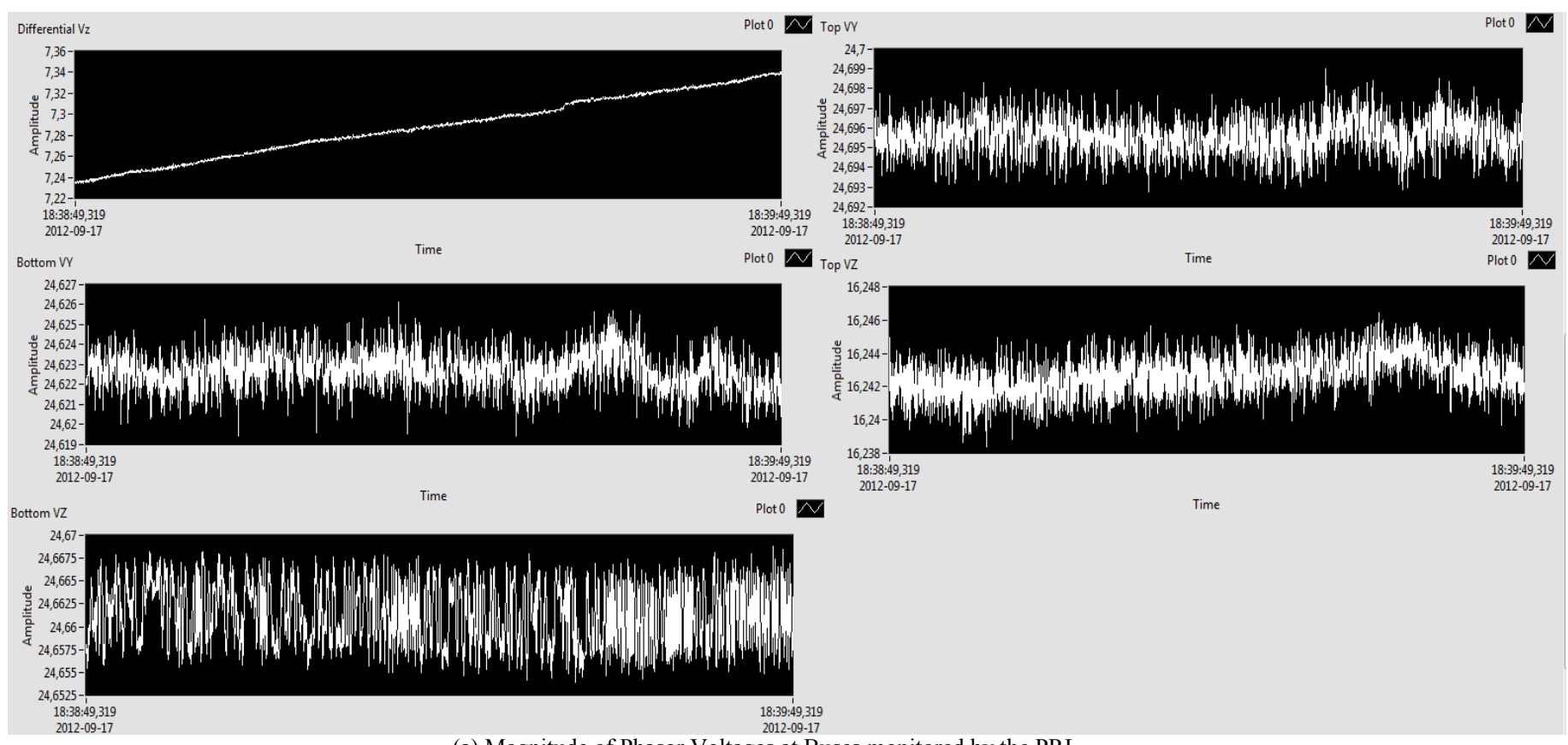

(a) Magnitude of Phasor Voltages at Buses monitored by the PRL

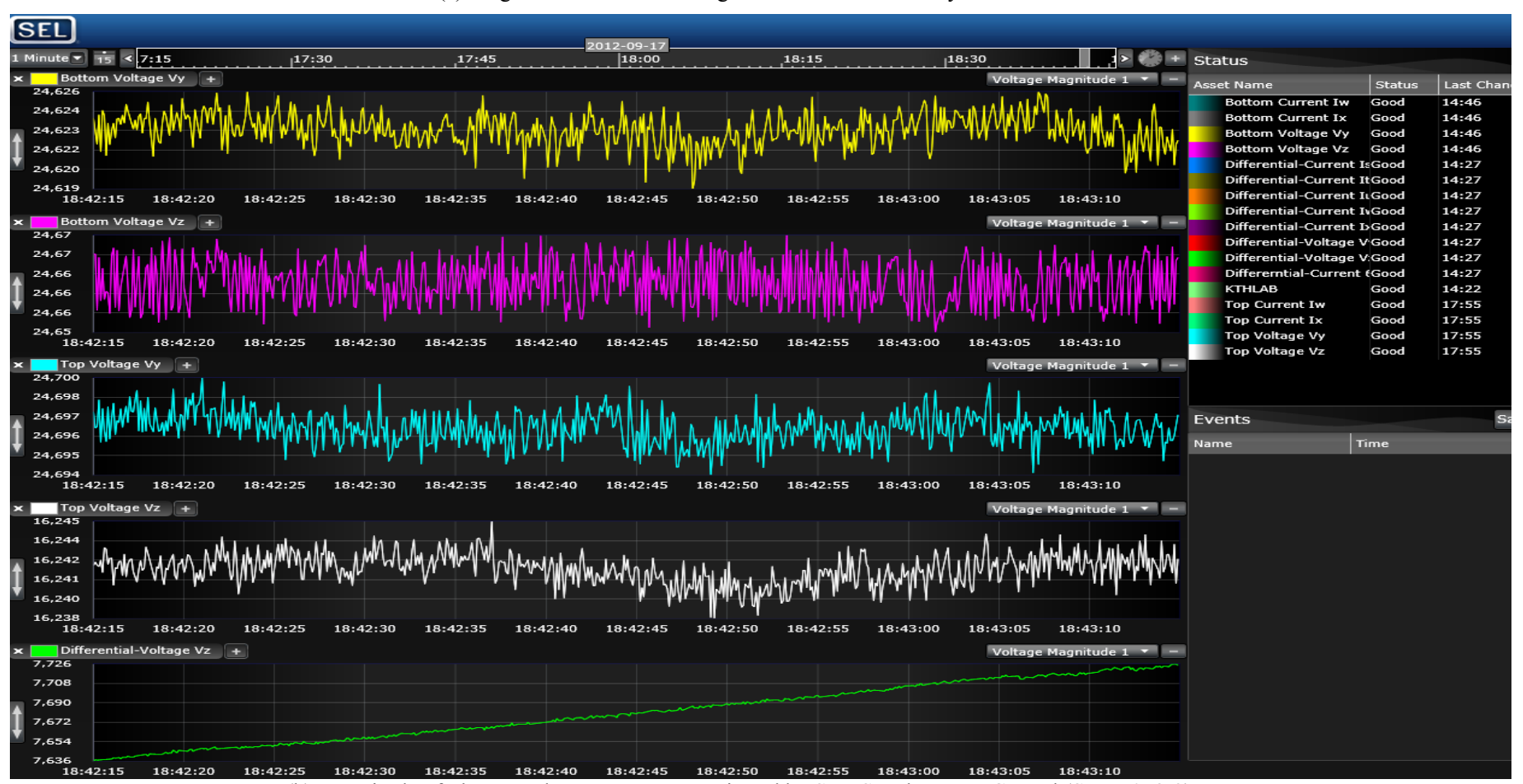

(b) Magnitude of Phasor Voltages at Buses monitored by SEL Synchrowave Central (SEL-5078-2)

Figure 12. Magnitude of Phasor Voltages at Buses monitored by the PRL and SEL Synchrowave Central (SEL-5078-2)

\section{SYNCHROPHASOR BASED CUSTOM APPLICATION DEVELOPMENT USING THE PRL SDK}

\section{A. Mode Meter Application}

The main objective of developing the PRL software development toolkit was testing an integral WAMS. To show one example of the development of WAMS applications, a real-time mode meter application (focusing on inter-area mode frequency estimation) was developed [13] [14]. The experimental setup and PMU data described in Section IV are used in order to estimate the frequency of the electromechanical modes of the power system. The application is tested in two experiments. In the first experiment, a power system is simulated in Opal-RT real-time simulator whereas for the second experiment PMUs are fed from the low-voltage network in laboratory facility. A user interface of the mode meter application is shown in Fig. 13 (case with real frequency measurements).

The developed mode meter uses spectral estimation algorithms in order to identify peaks in the spectrum and consequently frequency of the dominant electromechanical modes. Three different spectral estimators are used ensuring accurate signal spectrum estimation: (1) Welch's method, (2) Auto-Regressive (AR) method, and (3) Auto-Regressive Moving Average (ARMA) method. 


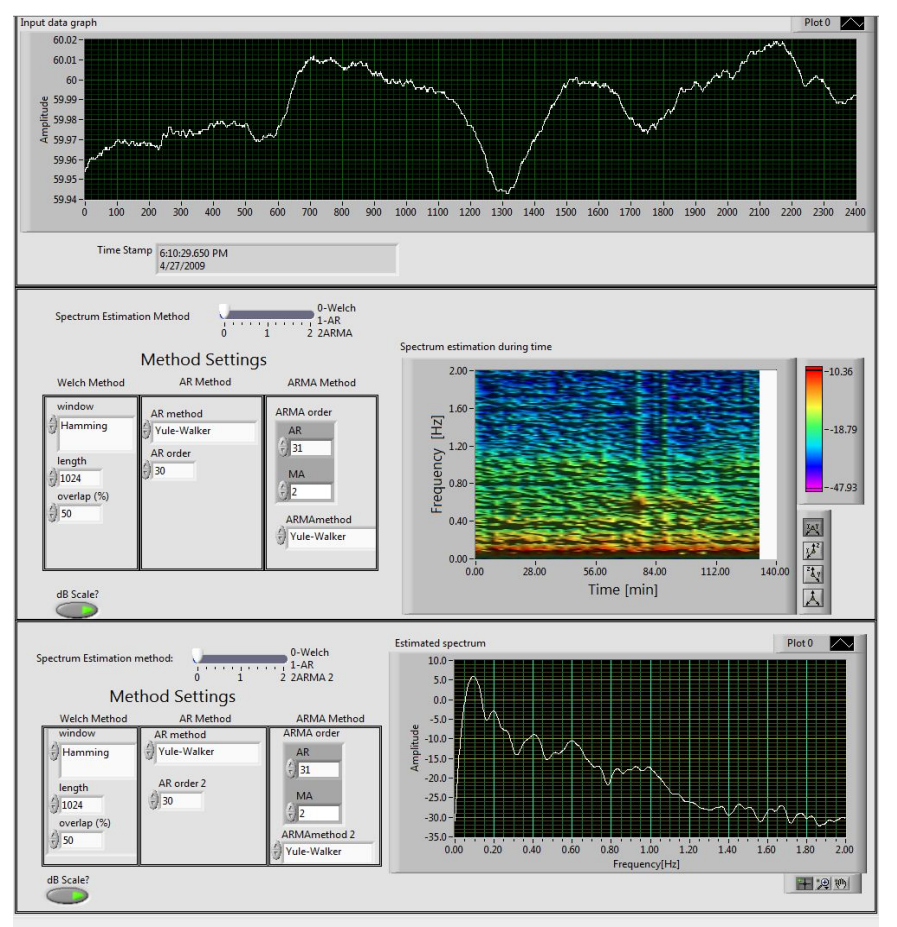

Figure 13. Labview mode meter application

The first method (Welch's) is a non-parametric method, meaning that there is no assumed underlying model of the measured signal. The second and third methods are parametric methods using AR and ARMA models of the measured signal, where AR model is a special case of the more general ARMA model.

The upper part of the mode meter interface shows measured signal in a time-moving panel (a frequency measurement signal in this case). The second part shows change of the spectrum during the time, what is referred to as a spectrogram. A single vertical line in the spectrogram is constructed from the single spectrum estimate and new vertical lines are successfully added every two minutes. The bottom part of the interface shows average spectrum computed using longer data records (up to two hours). This figure gives an insight into average behavior of the system.

\section{CONCLUISON}

Wide Area Monitoring Systems are very complex systems integrating different domains: from communication protocols to power system applications. This complexity makes difficult the full scale testing of such systems and consequently hinders further research and development of PMU applications.

This paper presents a Software Development Toolkit which makes full scale testing in real-time easier for researchers, liberating them of time consuming synchrophasor data handling. This SDK is based on IEEE C37.118.2 protocol, making this solution independent on any utilized equipment and their manufacturers. By using this SDK, a researcher can manipulate with synchrophasor data in LabView environment which enables fast software prototyping and testing for real-time applications.

To validate described approach, the test bench with four PMUs and real-time simulator was designed. The real-time simulator simulates the behavior of a 5 bus power system whose variables are measured by the PMUs. Further, the PMU outputs are imported in the LabView environment where simple mode meter application is developed. Presented experimental setup shows it is possible to design real-life hardware in the loop experiments with reasonable effort.

\section{ACKNOWLEDGEMENTS}

The donation of different hardware and technical support provided by Schweitzer Engineering Laboratories, ABB Substation Automation Products, Megger/Programma, and National Instruments, is greatly acknowledged.

M. Shoaib Almas is supported by Nordic Energy Research through the STRONg $g^{2}$ rid project. L. Vanfretti is supported by the STandUP for Energy collaboration initiative and Statnett SF, the Norwegian Transmission System Operator.

\section{REFERENCES}

[1] A. Phadke and R. de Moraes, "The Wide World of Wide-area Measurement," IEEE Power and Energy Magazine, vol. 6, no. 5, pp. 52 -65, Sept.-Oct. 2008

[2] V. Terzija et al., "Wide-Area Monitoring, Protection, and Control of Future Electric Power Networks," Proceedings of the IEEE, vol. 99, no. 1, pp. $80-93$, Jan. 2011.

[3] A. Leirbukt et al., "Deployment of a SCADA integrated wide area monitoring system," 2008 IEEE/PES Transmission and Distribution Conference and Exposition: Latin America, Aug. 2008.

[4] K. Uhlen et al., "Monitoring amplitude, frequency and damping of power system oscillations with PMU measurements," IEEE PES General Meeting 2008, July 2008.

[5] K. Uhlen, L. Vanfretti, et al., "Wide-Area Power Oscillation Damper Implementation and Testing in the Norwegian Transmission Network," IEEE PES General Meeting 2012, July 2012.

[6] R. Leelaruji, L. Vanfretti and M.S. Almas, "Voltage stability monitoring using sensitivities computed from synchronized phasor measurement data" IEEE PES General Meeting 2012, July 2012.

[7] C. Taylor et al., "WACS-Wide-Area Stability and Voltage Control System: R\&D and Online demonstration," Proceedings of the IEEE, vol. 93, no. 5, pp. 892 -906, May 2005.

[8] M. Begovic et al, "Wide-area protection and emergency control," Proceedings of the IEEE, vol. 93, no. 5, pp. 876-891, may 2005.

[9] L. Vanfretti, et al., "SmarTS Lab A Laboratory for Developing Applications for WAMPAC Systems", IEEE PES General Meeting 2012, July 2012.

[10] C37.118.2-2011 - IEEE Standard for Synchrophasor Data Transfer for Power System.

[11] E.O. Echweitzer, et al., "Real-time synchrophasor applications in power system control and protection", 10th IET International Conference on Development in Power System Protection (DPSP), Mar. 2010.

[12] V. Salehi, "Laboratory-Based Smart Power System, Part II: Control, Monitoring, and Protection," IEEE Transactions on Smart Grids, vol. 3, no. 3, pp. 1405-1417, Sept. 2012.

[13] A. Messina (Ed.), Inter-area oscillations in power systems: a nonlinear and nonstationary perspective, Springer Verlag, 2009.

[14] L. Vanfretti et al. "Application of ambient analysis techniques for the estimation of electromechanical oscillations from measured PMU data in four different power systems," European Transaction on Electrical Power, vol. 21, no. 4, pp. 1640-1656, May 2011. 